\title{
À propos du communiqué de l'Académie nationale de médecine
}

\author{
About the Press Release from the National Academy of Medicine \\ F. Guillemin - M. Schneider • au nom de l'ensemble du comité de la revue Oncologie \\ (C) Lavoisier SAS 2016
}

L'Académie nationale de médecine a fait un communiqué sur la valorisation des revues médicales en langue française suite à la démarche de la Conférence des présidents du Conseil national des universités d'introduire un score pédagogique dans l'épreuve de titres et travaux des candidats à une fonction universitaire.

Les soins, l'enseignement et la recherche sont les missions principales des universitaires. Ce ne sont pas les seules, et presque toujours, ils devront assumer, en plus, des charges de gestion, de management et d'animation d'équipe. Les critères à évaluer lors du passage devant le $\mathrm{CNU}$ sont donc très divers et complémentaires.

Une standardisation des modes d'évaluation est utile. Des règles d'évaluation claires et connues de tous simplifient considérablement la préparation des candidats et leur évaluation par les juges. Le score SIGAPS reflète assez bien l'activité « Recherche ». Le volet « Soins » est apprécié sur la base de l'activité individuelle de soins et sur la responsabilité d'animation d'un secteur hospitalier. Le chapitre « Enseignement » comporte des items allant des publications à caractère didactique jusqu'à l'implication pédagogique dans l'organisation d'enseignements de haut niveau pour les jeunes collègues, y compris dans le cadre de sociétés savantes, ou comme, plus simplement, pour délivrer des messages clairs et utiles aux correspondants de ville. Bien que des initiatives aient été prises par des CHU avec des écoles de commerce pour former de futurs leaders médecins, les aspects managériaux au sens large ne sont que peu pris en considération.

Le score SIAPS devrait rendre service s'il est équilibré et ne fait pas un amalgame de publications ayant des qualités d'information et de pédagogie très variables. Les règles de construction du score doivent être simples et applicables pour toutes les sous-sections.

Il n'en reste pas moins vrai que la valeur humaine du candidat ne s'apprécie pas par un score. Cette dimension humaine se traduit par l'enthousiasme et le charisme, par l'imagination et la rigueur, par la générosité et l'empathie et par bien d'autres qualités parfois inattendues qui sont la richesse de la personnalité de ceux qui feront la médecine de demain.

\footnotetext{
F. Guillemin $(\bowtie) \cdot$ M. Schneider $(\bowtie)$

Sous-section 47-02 «Cancérologie ; radiothérapie »

$\mathrm{du}$ Conseil national des universités (CNU)

e-mail : francois.guillemin@reims.unicancer.fr,

maurice.schneider@unice.fr

F. Guillemin

Institut de cancérologie Jean-Godinot,

centre de lutte contre le cancer de Reims,

Champagne-Ardenne, 1, rue du Général-Kœnig, CS 80014,

F-51726 Reims cedex, France

M. Schneider

Comité des Alpes-Maritimes de la Ligue contre le cancer,

3, rue Alfred-Mortier, F-06000 Nice, France
} 